\title{
Criação de uma Nova Transformada Wavelet Aplicada ao Reconhecimento Digital de Padrões em Sinais Neurais do Campo Visual da Mosca Diptera $^{1}$
}

R.C. GUIDO ${ }^{2}$, J.F.W. SLAETS ${ }^{3}$, L.O.B. ALMEIDA ${ }^{4}$, Grupo de Instrumentação e Informática. Instituto de Física de São Carlos - USP, Avenida Trabalhador São Carlense 400, 13560-970 São Carlos, SP, Brasil.

\begin{abstract}
Resumo: O presente trabalho apresenta uma técnica para criação de uma transformada wavelet, aqui chamada de SPIKELET-4, otimizada para o reconhecimento de alguns formatos de spikes (picos) e overlaps (sobreposições de picos) contidos num sinal digitalizado originado do neurônio H1, sensível aos estímulos visuais do meio externo, do cérebro de uma mosca Diptera. Toda a técnica para criação da transformada, que está associada a um algoritmo especialmente projetado para ser usado em conjunto com ela, está demonstrada com detalhes e possui resultados que não foram alcançados com nenhuma outra transformada wavelet, nem mesmo com a transformada de Daubechies.
\end{abstract}

\section{Introdução}

Existe uma série de artigos atualmente, tais como [3], [6] e [2], que descrevem técnicas para criar transformadas wavelet otimizadas para o reconhecimento de um determinado formato de sinal. Em especial, a motivação do presente trabalho surgiu de uma série de testes, sem muito sucesso, realizados com diversas wavelets conhecidas (Daubechies, Coiflets, Symmlets e outras), no sentido de encontrar uma transformada e um algoritmo do tipo tempo-frequência, de baixa complexidade computacional, que possibilitasse a distinção de, pelo menos, 6 tipos de padrões encontrados em um sinal proveniente do neurônio $H 1$ do cérebro de uma mosca Diptera [14], recolhido de maneira etracelular, com o auxílio de um eletrodo, quando ela recebe estímulos visuais do meio externo.

A Figura 1 exibe os 6 tipos de sinais em questão, que são classificados respectivamente, da esquerda para a direita, de overlap acentuado à esquerda, overlap à esquerda, spike, overlap à direita, overlap acentuado à direita e overlap à esquerda

\footnotetext{
${ }^{1}$ Desenvolvido no Dipteralab - IFSC - USP (http://www.dipteralab.if.sc.usp.br).

${ }^{2}$ guido@if.sc.usp.br; autor da tese de doutorado à qual o presente trabalho está ligado.

3 jan@if.sc.usp.br; orientador

${ }^{4}$ lirio@if.sc.usp.br; técnico colaborador do presente trabalho
} 
$e$ à direita. Como cada um dos overlaps mencionados são sobreposições de spikes em diversas posições, a SPIKELET-4 (wavelet criada) será otimizada para corresponder com um spike, aqui chamado spike médio padrão, de forma que os overlaps sejam identificados através da localização dos spikes que os compõe.

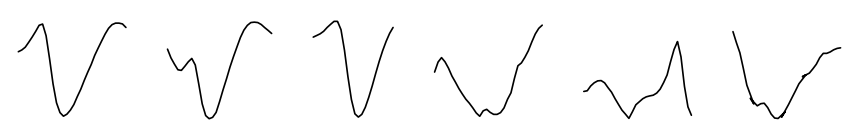

Figura 1: Os 6 padrões de formatos procurados no sinal da mosca.

\section{Obtenção do spike médio padrão}

O spike médio padrão foi determinado, por um processo experimental, como sendo a curva média entre 80 spikes pré-selecionados. A Figura 2, a seguir, exibe este sinal de interesse e os valores numéricos respectivos $(x=$ amostra, $y=$ amplitude $)$ para cada um dos 32 pontos que o definem.
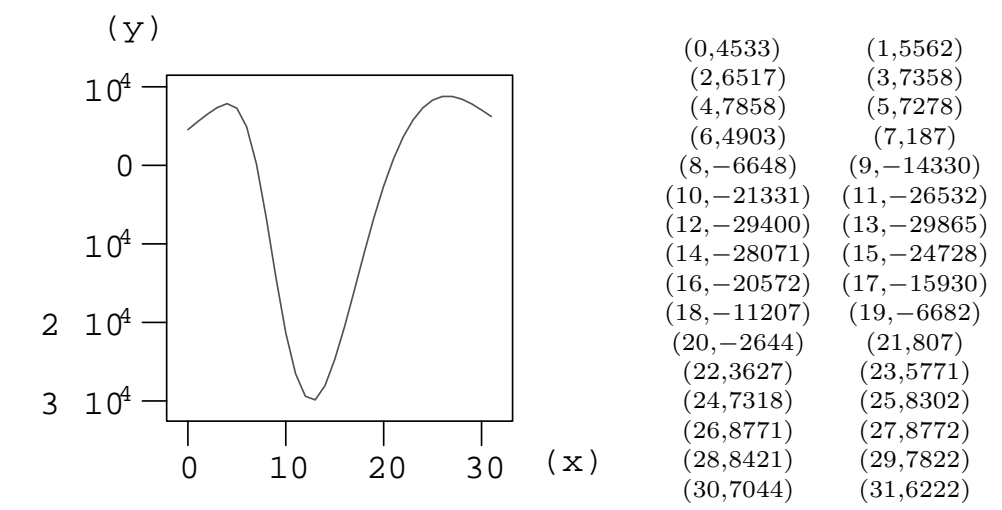

Figura 2: spike médio padrão

\section{Construção da SPIKELET-4}

Depois de definido o sinal de interesse (spike médio padrão), três conjuntos de etapas foram seguidas: as etapas do grupo A (A1, A2 e A3 - transformada de Daubechies), as etapas do grupo B (B1, B2 e B3 - maximizacao de energia), que ocorrem em paralelo com as etapas do grupo A, e, finalmente, as etapas do grupo C (C1, C2 e C3 - obtenção dos coeficientes) que são posteriores às etapas dos grupos A e B. O processo está descrito detalhadamente a seguir, em cada uma das suas etapas: 


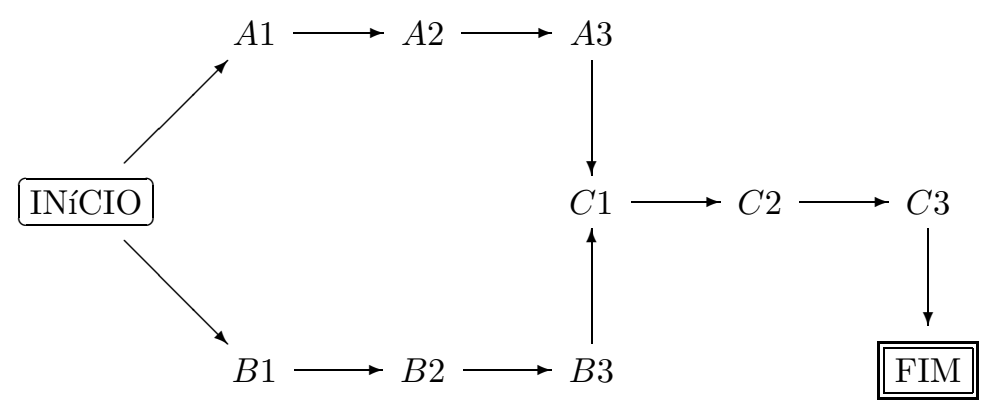

- Etapa A1: os três grupos de equações logo a seguir, quando reunidos de modo a formar um sistema nas incógnitas $h_{0}, \ldots, h_{n}$, correspondem definição da transformada de Daubechies

$$
\sum_{k=0}^{n-1}(-1)^{k} h_{k} k^{b}=0 \quad, \quad \sum_{k=0}^{n-1} h_{k}=2 \quad, \quad \sum_{k=0}^{n-1} h_{k+2 l}=2 \delta_{0, l},
$$

onde $\left(b=0,1, \ldots, \frac{n}{2}-1\right)$ e $(l \in Z)$. O primeiro grupo é o responsável por fazer com que a função scaling [13] possa representar um polinômio de grau até, mas não maior que, $\frac{n}{2}$, sendo $n$ o tamanho do suporte do filtro. Isso implica que a wavelet possui $\frac{n}{2}$ momentos nulos [15]. O segundo grupo, constituído por uma única equação, cuida da normalização dos coeficientes e vem do fato de que para a definição das funções scaling de um dado formato, a área correspondente embaixo de cada curva deve ser unitária: $\int \Phi(x) d x=1$. O terceiro grupo, cuida da ortogonalidade entre cada par de vetores da função scaling.

- Etapa A2: nesta etapa, retira-se a condição de ortogonalidade da transformada de Daubechies, já que esta condição não terá uso na transformada SPIKELET-4

$$
\sum_{k=0}^{n-1}(-1)^{k} h_{k} k^{b}=0 \quad, \quad \sum_{k=0}^{n-1} h_{k}=2 .
$$

- Etapa A3: as equações da etapa A2 são especificadas para um suporte $n=$ 4. O sistema resultante desta etapa é linear e possui $\frac{n}{2}+1=3$ equações com $n=4$ incógnitas

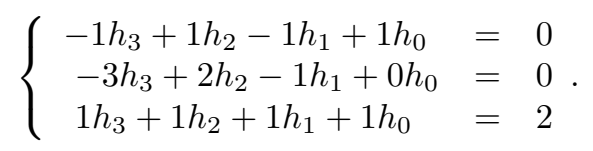

- Etapa B1: realiza-se a convolução seguida de sub-amostragem por 2, do spike médio padrão com um filtro de suporte 4 com coeficientes genéricos $h_{0}, \ldots, h_{3}$ (incógnitas). O sinal resultante é a projeção spike médio padrão (designado doravante de vetor $\vec{u}$ ) num subespaço vetorial $W$. 
- Etapa B2: a energia do sinal resultante da etapa B1 anterior (projeção de $\vec{u}$ em $W$ ) é maximizada, objetivando transferir o máximo de energia do sinal original para o sinal resultante. Para isso, são calculadas as suas derivadas parciais em relação a cada um dos coeficientes

$$
\partial \frac{\overrightarrow{E\left(P_{w} U\right)}}{\partial h_{0}}=0 \quad, \quad \partial \frac{\overrightarrow{E\left(P_{w} U\right)}}{\partial h_{1}}=0 \quad, \quad \partial \frac{\overrightarrow{E\left(P_{w} U\right)}}{\partial h_{3}}=0 \quad, \quad \partial \frac{\overrightarrow{E\left(P_{w} U\right)}}{\partial h_{3}}=0 .
$$

- Etapa B3: cada uma das 4 derivadas parciais obtidas na etapa B2 é igualada a 0 , formando um sistema linear homogêneo de 4 equações com 4 incógnitas

$$
\left\{\begin{array}{rl}
-3.4984 h_{3}+3.3876 h_{2}-3.1079 h_{1}+2.6457 h_{0} & =0 \\
-3.3876 h_{3}+3.4876 h_{2}-3.3967 h_{1}+3.0924 h_{0} & =0 \\
-3.1079 h_{3}+3.3967 h_{2}-3.5100 h_{1}+3.4000 h_{0} & =0 \\
-2.6457 h_{3}+3.0924 h_{2}-3.4000 h_{1}+3.5006 h_{0} & =0
\end{array} .\right.
$$

- Etapa C1: reúnem-se as equações obtidas nas etapas A3 e B3. O novo sistema é linear e possui $\left(\frac{n}{2}+1\right)+n=7$ equações e $n=4$ incógnitas

$$
\left(\begin{array}{cccc}
-3.4984 & 3.3876 & -3.1079 & 2.6457 \\
-3.3876 & 3.4876 & -3.3967 & 3.0924 \\
-3.1079 & 3.3967 & -3.5100 & 3.4000 \\
-2.6457 & 3.0924 & -3.4000 & 3.5006 \\
-1 & 1 & -1 & 1 \\
-3 & 2 & -1 & 0 \\
1 & 1 & 1 & 1
\end{array}\right)\left(\begin{array}{c}
h_{0} \\
h_{1} \\
h_{2} \\
h_{3}
\end{array}\right)=\left(\begin{array}{l}
0 \\
0 \\
0 \\
0 \\
0 \\
0 \\
2
\end{array}\right)
$$

- Etapa C2: devido inexistência de solução exata para o sistema da etapa C1, optou-se pela solução através do Método dos Mínimos Quadrados [4]. O sistema é, então, expresso na forma matricial e cada lado da igualdade é multiplicado pela matriz transposta dos pesos das incógnitas

$$
\begin{gathered}
\left(\begin{array}{ccccccc}
-3.4984 & -3.3876 & -3.1079 & -2.6457 & -1 & -3 & 1 \\
3.3876 & 3.486 & 3.3967 & 3.0924 & 1 & 2 & 1 \\
-3.1079 & -3.3967 & -3.5100 & -3.4000 & -1 & -1 & 1 \\
2.6457 & 3.0924 & 3.4000 & 3.5006 & 1 & 0 & 1
\end{array}\right)\left(\begin{array}{ccccc}
-3.4984 & 3.3876 & -3.1079 & 2.6457 \\
-3.3876 & 3.4876 & -3.3967 & 3.0924 \\
-3.1079 & 3.3967 & -3.5100 & 3.4000 \\
-2.6457 & 3.0924 & -3.4000 & 3.5006 \\
-1 & 1 & -1 & 1 \\
-3 & 2 & -1 & 0 \\
1 & 1 & 1 & 1
\end{array}\right)\left(\begin{array}{c}
h_{0} \\
h_{1} \\
h_{2} \\
h_{3}
\end{array}\right) \\
=\left(\begin{array}{ccccccc}
-3.4984 & -3.3876 & -3.1079 & -2.6457 & -1 & -3 & 1 \\
3.3876 & 3.4876 & 3.3967 & 3.0924 & 1 & 2 & 1 \\
-3.1079 & -3.3967 & -3.5100 & -3.4000 & -1 & -1 & 1 \\
2.6457 & 3.0924 & 3.4000 & 3.5006 & 1 & 0 & 1
\end{array}\right)\left(\begin{array}{l}
0 \\
0 \\
0 \\
0 \\
0 \\
0 \\
2
\end{array}\right) .
\end{gathered}
$$

- Etapa C3: a solução obtida implica nos coeficientes $h_{0}, \ldots, h_{3}$ encontrados, que representam um filtro passa-baixa. Como o sistema é definido do tipo QMF ( Quadrature Mirror Filter) [7], os coeficientes do filtro passa-alta respectivo obedecem a equação $g_{n}=(-1)^{n} h_{3-n}, \operatorname{com} n=0, \ldots, 3$, ficando, portanto, a SPIKELET-4 assim definida:

$$
\left\{\begin{array}{cll}
h_{0}=0.26964482896235847376 & , & g_{0}=0.23524044702452745481 \\
h_{1}=0.76237548312490721614 & , & g_{1}=-0.73270322306815560687 \\
h_{2}=0.73270322306815560687 & , & g_{2}=0.76237548312490721614 \\
h_{3}=0.23524044702452745481 & , & g_{3}=-0.26964482896235847376
\end{array} .\right.
$$

A Figura 3 exibe a resposta em frequência do filtro passa-baixa SPIKELET-4. De posse dos coeficientes dos filtros, podemos construir as funções scaling e wavelet. 
(y)

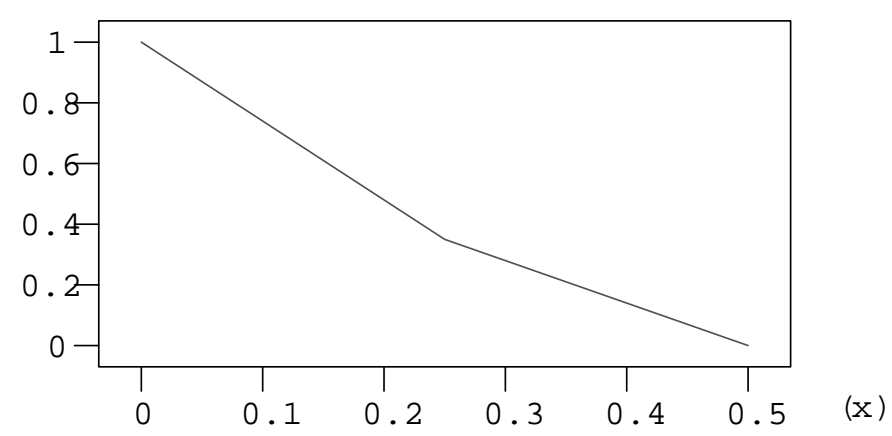

Figura 3: Resposta em frequência do filtro passa-baixa SPIKELET-4, onde $x$ representa a frequência angular $(0.5=\pi)$ e $y$ representa a amplitude.

\subsection{Função Scaling}

De acordo com a teoria das wavelets, a função scaling é definida recursivamente pela equação de dilatação e translação $\Phi(n)=\sum_{k} h_{k} \Phi(2 n-k)$. Se temos $n=$ 4 coeficientes, os valores de $\Phi(x)$ para $x<0$ são nulos. Da mesma forma, para $x>n-1$, também a função é nula. Sendo assim, existirão valores, possivelmente diferentes de 0 , para $\Phi(0), \Phi(1), \Phi(2), \Phi(3)$ e, também, nos pontos intermediários $\Phi\left(\frac{1}{2}\right), \Phi\left(\frac{3}{2}\right)$ e $\Phi\left(\frac{5}{2}\right)$. De acordo com a definição recursiva dada acima, temos, então

$$
\left\{\begin{array}{l}
\Phi(0)=h_{0} \Phi(0) \\
\Phi(1)=h_{0} \Phi(2)+h_{1} \Phi(1)+h_{2} \Phi(0) \\
\Phi(2)=h_{1} \Phi(3)+h_{2} \Phi(2)+h_{3} \Phi(1) \\
\Phi(3)=h_{3} \Phi(3)
\end{array}\right.
$$

Na forma matricial, isso é, $M T=T$ onde $M=\left(\begin{array}{cccc}h_{0} & 0 & 0 & 0 \\ h_{2} & h_{1} & h_{0} & 0 \\ 0 & h_{3} & h_{2} & h_{1} \\ 0 & 0 & 0 & h_{3}\end{array}\right)$ e $T=\left(\begin{array}{c}\Phi(0) \\ \Phi(1) \\ \Phi(2) \\ \Phi(3)\end{array}\right)$.

Portanto, a matriz $\mathrm{T}$ com os valores da função scaling procurada é o autovetor de $\mathrm{M}$ correspondente ao autovalor 1 . Como a solução do $\operatorname{problema}(M-I) t=0$ não é única, uma condição de normalização é necessária para determinar um único autovetor. Esta condição é: $\sum_{k} \Phi(k)=1$. Sendo assim, temos

$$
\left\{\begin{array}{l}
\left(h_{0}-1\right) \Phi(0)=0 \\
h_{2} \Phi(0)+\left(h_{1}-1\right) \Phi(1)+h_{0} \Phi(2)=0 \\
h_{3} \Phi(1)+\left(h_{2}-1\right) \Phi(2)+h_{1} \Phi(3)=0 \\
\left(h_{3}-1\right) \Phi(3)=0 \\
\Phi(0)+\Phi(1)+\Phi(2)+\Phi(3)=1
\end{array}\right.
$$

Como já encontramos os valores de $h_{0}, \ldots h_{3}$, e eles são todos diferentes de zero, o sistema acima implica em: 


$$
\left\{\begin{array}{l}
\Phi(0)=\Phi(3)=0 \\
\left(h_{1}-1\right) \Phi(1)+h_{0} \Phi(2)=0 \\
h_{3} \Phi(1)+\left(h_{2}-1\right) \Phi(2)=0 \\
\Phi(0)+\Phi(1)+\Phi(2)+\Phi(3)=1
\end{array}\right.
$$

e, portanto,

$$
\left\{\begin{array}{l}
-0.23762451687509278386 \Phi(1)+0.26964482896235847376 \Phi(2)=0 \\
0.23524044702452745481 \Phi(1)-0.26729677693184439313 \Phi(2)=0 \\
\Phi(1)+\Phi(2)=1
\end{array} .\right.
$$

Devido ao fato de que as equações da etapa A3 anterior foram perturbadas pelo ajuste via Mínimos Quadrados realizado para encontrar os coeficientes $h_{0}, \ldots, h_{3}$, as duas primeiras equações que formam o sistema logo acima não são exatamente múltiplas uma da outra, como ocorre na criação da função scaling do filtro de Daubechies. Caso tal propriedade estivesse presente, o sistema em questão possuiria solução exata e diferente de 0 . Optou-se, então, pela solução utilizando novamente o Método dos Mínimos Quadrados, sendo obtido, portanto,

$$
\Phi(1)=0.53172639832546342298 \quad, \quad \Phi(2)=0.46827358753833858707 .
$$

e, para os pontos intermediários, $\Phi\left(\frac{x}{2}\right)=\sum_{k} h_{k} \Phi(x-k)$. Daí,

$$
\begin{aligned}
& \Phi\left(\frac{1}{2}\right)=h_{0} \Phi(1)=0.14337727373124048436 \\
& \Phi\left(\frac{3}{2}\right)=h_{1} \Phi(2)+h_{2} \Phi(1)=0.74659794837766346731 \\
& \Phi\left(\frac{5}{2}\right)=h_{3} \Phi(2)=0.11015688806229795293
\end{aligned}
$$

\subsection{Função wavelet}

Utilizando os resultados obtidos no cálculo da função scaling e a definição recursiva da função wavelet $\Psi(n)=\sum_{k} g_{k} \Phi(2 n-k)$, obtemos

$$
\left\{\begin{array}{l}
\Psi(0)=g_{0} \Phi(0) \\
\Psi(1)=g_{0} \Phi(2)+g_{1} \Phi(1)+g_{2} \Phi(0) \\
\Psi(2)=g_{1} \Phi(3)+g_{2} \Phi(2)+g_{3} \Phi(1) \\
\Psi(3)=g_{3} \Phi(3)
\end{array}\right.
$$

o que leva a

$$
\left\{\begin{array}{l}
\Psi(0)=\Psi(3)=0 \\
\Psi(1)=g_{0} \Phi(2)+g_{1} \Phi(1)=-0.27944075778119104037 \\
\Psi(2)=g_{2} \Phi(2)+g_{3} \Phi(1)=0.21362302880293393414
\end{array}\right.
$$

e, para os pontos intermediários, $\Psi\left(\frac{x}{2}\right)=\sum_{k} g_{k} \Phi(x-k)$. Portanto,

$$
\begin{aligned}
& \Psi\left(\frac{1}{2}\right)=g_{0} \Phi(1)=0.12508355563682396761 \\
& \Psi\left(\frac{3}{2}\right)=g_{1} \Phi(2)+g_{2} \Phi(1)=0.06226960294661321171 \\
& \Psi\left(\frac{5}{2}\right)=g_{3} \Phi(2)=-0.12626755141936529814
\end{aligned}
$$


(y)

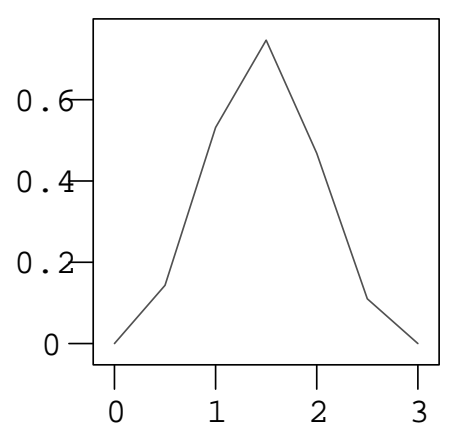

(y)

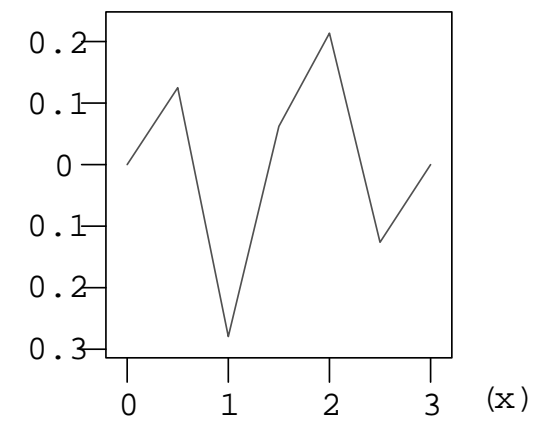

Figura 4: Funções scaling e wavelet da transformada SPIKELET-4.

A Figura 4 exibe os gráficos das funções scaling e wavelet da SPIKELET-4.

\subsection{Análise dos Momentos Nulos da SPIKELET-4}

A transformada de Daubechies de suporte 4 possui, por construção, 2 momentos nulos. Embora a transformada SPIKELET-4 conserve esta equação até a etapa C1, o ajuste por Mínimos Quadrados utilizado na etapa C2, causa uma perturbação nos momentos. O cálculo de um momento $M$ de ordem $m$, usando a definição $\left(\sum_{t} t^{m} \Psi(t), \operatorname{com} t=0, \frac{1}{2}, 1, \ldots, \frac{5}{2}, 3\right)$, mostra que, embora eles não estejam demasiadamente distantes de 0, a SPIKELET-4 não obedece a nenhuma condição de momento: momento de ordem $\mathbf{0}=-0.0047321$ e momento de ordem $\mathbf{1}=-0.0119173$.

\subsection{Estabilidade e Causalidade da SPIKELET-4}

Tomando os coeficientes da SPIKELET-4, é possível escrever uma equação de diferenças do filtro correspondente que represente o sistema e, em seguida, obter a sua Função de Transferência, com o uso da transformada Z Im $^{[8] \text {. }}$

O Diagrama de Bode correspondente está na Figura 5, onde os círculos correspondem aos zeros e a cruz corresponde aos pólos da função de transferência. O fato de todos os pólos estarem no centro do círculo unitário implica que o sistema é estável e causal. Como existem dois zeros fora do círculo unitário, o sistema inverso não é causal e estável, entretanto, como ele não é necessário para a aplicação do algoritmo proposto neste trabalho, e nem mesmo foi calculado aqui, este fato não causa problemas.

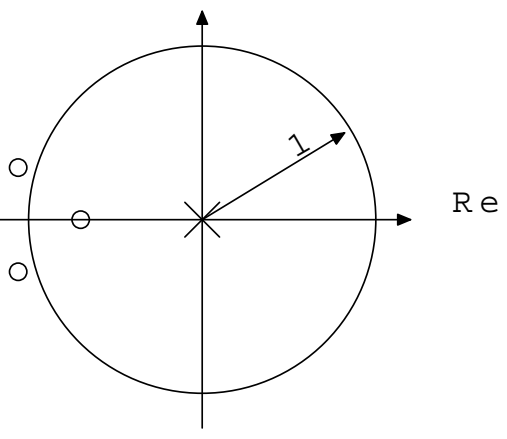

Figura 5: Diagrama de Bode. 


\subsection{Considerações}

Para todos os testes foi utilizada precisão numérica de 20 casas decimais nos coeficientes do filtro. Em especial, a SPIKELET-4 possui as seguintes características:

- wavelet não simétrica, com filtro tipo FIR (Finite Impulse Response [9] ) de suporte compacto $=4$, estável, causal e com resposta em fase não linear;

- energia $=\sum_{k=0}^{n-1}\left(h_{k}\right)^{2}=1.24611680 \neq 1 \mathrm{e}$, portanto, não há conservação da energia do sinal original aplicado à transformada;

- cada vetor $V_{j}$ é ortogonal apenas ao vetor $B_{j}, j=0,1, \ldots$, onde $\{V\}$ são os vetores de suporte 4 gerados pela função scaling e $\{B\}$ são aqueles gerados pela função wavelet;

- a maior amplitude da transformada aplicada num spike de 32 amostras é obtida nos pontos que estão relacionados com a borda de subida (pontos entre o pico e o fim) do sinal [5]. Esse fato é de fundamental importância para o algoritmo a seguir.

\section{Algoritmo de reconhecimento dos padrões}

O algoritmo associado a SPIKELET-4 é bastante simples, possui ordem de complexidade linear e consiste apenas em analisar a transformada nível 3 do sinal de 32 amostras (a mesma quantidade do spike médio padrão) que se queira classificar [5]. Como o sinal sob análise é amostrado a uma taxa de $44100 K h z$, a máxima frequência presente é de $22050 \mathrm{Khz}$, devido ao Teorema de Nyquist [11] e, portanto, a decomposição implica em:

- amostras 0 a 3 da SPIKELET-4: descrevem a faixa de frequências de 0 a $2756,25 \mathrm{Khz}$ com incerteza de 8 pontos em relação ao sinal sob análise, devido a sub amostragem por 2 realizada a cada nível de decomposição;

- amostras 4 a 7 da SPIKELET-4: descrevem a faixa de frequências de 2756,25 a $5512,50 \mathrm{Khz}$ com incerteza de 8 pontos em relação ao sinal sob análise, devido a sub amostragem por 2 realizada a cada nível de decomposição.

Estas 2 sub-bandas (amostras 0 a 3 e 4 a 7) são suficientes para a análise, já que a decomposição de um spike/overlap nestas frequências mostra todas as bordas de subida existentes (região onde a SPIKELET-4 apresenta maior amplitude). Daí, de acordo com [1], para uma wavelet não simétrica, como é o caso da SPIKELET-4, as bordas de subida no sinal original correspondem aos pontos onde a transformada assume valor 0 (zero-crossings) e, consequêntemente, às inversões do sinal entre os seus pontos vizinhos. Especificamente, as inversões de sinal entre as amostras da SPIKELET-4 de um provável spike/overlap são:

- overlap acentuado à esquerda: inverte entre 1 e 2, 4 e 5;

- overlap à esquerda: inverte entre 1 e 2,4 e 5, 5 e 6 ; 
- spike: inverte entre 1 e 2, 4 e 5, 5 e 6,6 e 7 ;

- overlap à direita: inverte entre 1 e 2, 5 e 6,6 e 7;

- overlap acentuado à direita: inverte entre 1 e 2,6 e 7 ;

- overlap à esquerda e à direita: inverte entre 1 e 2, 4 e 5, 6 e 7;

- qualquer outra combinação implica que o sinal sob análise está fora dos padrões procurados.

\section{Testes, Resultados e Conclusões}

A transformada SPIKELET-4 e o algoritmo acima mostraram-se muito eficientes para a identificação da classe de sinais estudados e podem ser perfeitamente adaptados para outros sinais. O algoritmo possui complexidade linear, foi implementado na linguagem de programação $\mathrm{C}++$ em ambiente LINUX e, também, num DSP Digital Signal Processor ADSL21065L - SHARC da Analog Devices Inc, para uso em tempo-real. Em particular, nenhuma outra transformada wavelet associada a este algoritmo, ou a qualquer outro algoritmo para classificação de spikes/overlaps descrito na literatura [12], [10], foi capaz de distinguir todos os 6 tipos de sinais:

- Daubechies de suporte 4: distingue apenas 4 casos;

- Daubechies de suporte 20: distingue apenas 2 casos;

- Haar (suporte 2): distingue apenas 4 casos;

- Coiflet de suporte 6: distingue apenas 5 casos;

- Symmlet de suporte 8: distingue apenas 3 casos;

- Batlle-Lemariè de suporte 12: distingue apenas 5 casos;

- Beylkin de suporte 18: distingue apenas 4 casos;

- Vaidyanathan de suporte 24: distingue apenas 4 casos.

Um fato interessante é que, apenas com suporte 4, o algoritmo já apresentou funcionamento ótimo. As SPIKELETs com suportes maiores que 4 foram testadas, mas não apresentaram bons resultados, devido ao fato de que as equações das etapas A3 e B3 anteriores ficam muito discrepantes e, portanto, o ajuste por Mínimos Quadrados realizado na etapa C1 as perturba demais.

\footnotetext{
Abstract: This work aims to describe a technique to create a new wavelet transform, called SPIKELET-4, optimezed to spike and overlap pattern recognition, contained in a digitalized signal that comes from $\mathrm{H} 1$, which is the visual action potencial neuron of a Diptera's brain. The technique to construct the transform and the associated algorithm that works together with it, are detailed here. The results obtained show an improvement on all others wavelet transforms, including the Daubechies Transform.
} 


\section{Referências}

[1] P.S. Addison, "The Illustrated Wavelet Transform Handbook: Introductory Theory and Applications in Science, Engineering, Medicine and Finance", Institute of Physics Publishing, Edinburgh, 2002.

[2] M. Akay, "Time Frequency and wavelets in Biomedical Signal Processing", IEEE Press in Biomedical Engineering, New York, 1998.

[3] J.O. Chapa e R.M. Rao, Algoritms for Designing wavelets to Match a Specified Signal, IEEE Transactions on Signal Processing, 48, No. 12 (2000), 3395-3406.

[4] D.M. Claudio e J.M. Marins, "Cálculo Numérico Computacional: Teoria e Prática", 3. ed, Atlas, São Paulo, 2000.

[5] R.C. Guido, "Spikelet: uma nova transformada wavelet aplicada ao reconhecimento computacional de padrões, em tempo-real, de spikes e overlaps em sinais neurofisiológicos do campo visual da mosca", Tese de Doutorado, Instituto de Física de São Carlos - USP, São Carlos, SP, Brasil, 2003.

[6] A. Gupta, S.D. Joshi e S. Prasad, On a New Approach for Estimating Wavelet Matched to Signal, Depto of Computer Engineering Pub., Netaji Subhas Institute of Technology, Vol. 1, 2001.

[7] R.W. Hamming, "Digital Filters", 3.ed., Dover Publications, New York, 1989.

[8] S. Haykin e B.V. Veen, "Sinais e Sistemas", Bookman, Porto Alegre, 2001.

[9] J. Lane, J. Datta, B. Karley e J.Norwood, "DSP Filters", Prompt Publications, Indianapolis, 2001.

[10] M.S. Lewicki, A review of methods for spike sorting: the detection and classification of neural action potencials, Network Computing Neural Systems, 9 (1998), 53-78.

[11] A.V. Oppenhein e R.W. Schafer, "Discrete Time Signal Processing", 2. ed., Prentice Hall, New York, 1999.

[12] D. Rinberg, W. Bialek, H. Davidowitz e N.Tishby, Spike sorting in the frequency domain with overlap detection, NEC Research Institute Publ., 1 (2003), $1-40$.

[13] G. Strang, T. Nguyen, "Wavelets and Filter Banks", Wellesley-Cambridge Press, Wellesley, 1997.

[14] R.R.R.V. Steveninck, G.D. Lewen, S.P. Strong, R. Koberle e W. Bialek, Reproducibility and Variability in Neural Spike Trains, Science, 275 (1997), 18051808.

[15] J.R. Williams e K. Amaratunga, Introduction to Wavelets in Engineering, Int. Journal for Numerical Methods in Engineering, 37 (1994), 2365-2388. 